This item was submitted to Loughborough's Research Repository by the author.

Items in Figshare are protected by copyright, with all rights reserved, unless otherwise indicated.

\title{
Cross organisational compatible plans generation framework
}

PLEASE CITE THE PUBLISHED VERSION

http://dx.doi.org/10.1007/978-1-4471-2318-7_17

PUBLISHER

(C) Springer Verlag

VERSION

AM (Accepted Manuscript)

LICENCE

CC BY-NC-ND 4.0

REPOSITORY RECORD

Saleem, Mohammad, Paul Wai Hing Chung, Shaheen Fatima, and Wei Dai. 2019. "Cross Organisational Compatible Plans Generation Framework". figshare. https://hdl.handle.net/2134/11191. 
This item was submitted to Loughborough's Institutional Repository (https://dspace.lboro.ac.uk/) by the author and is made available under the following Creative Commons Licence conditions.

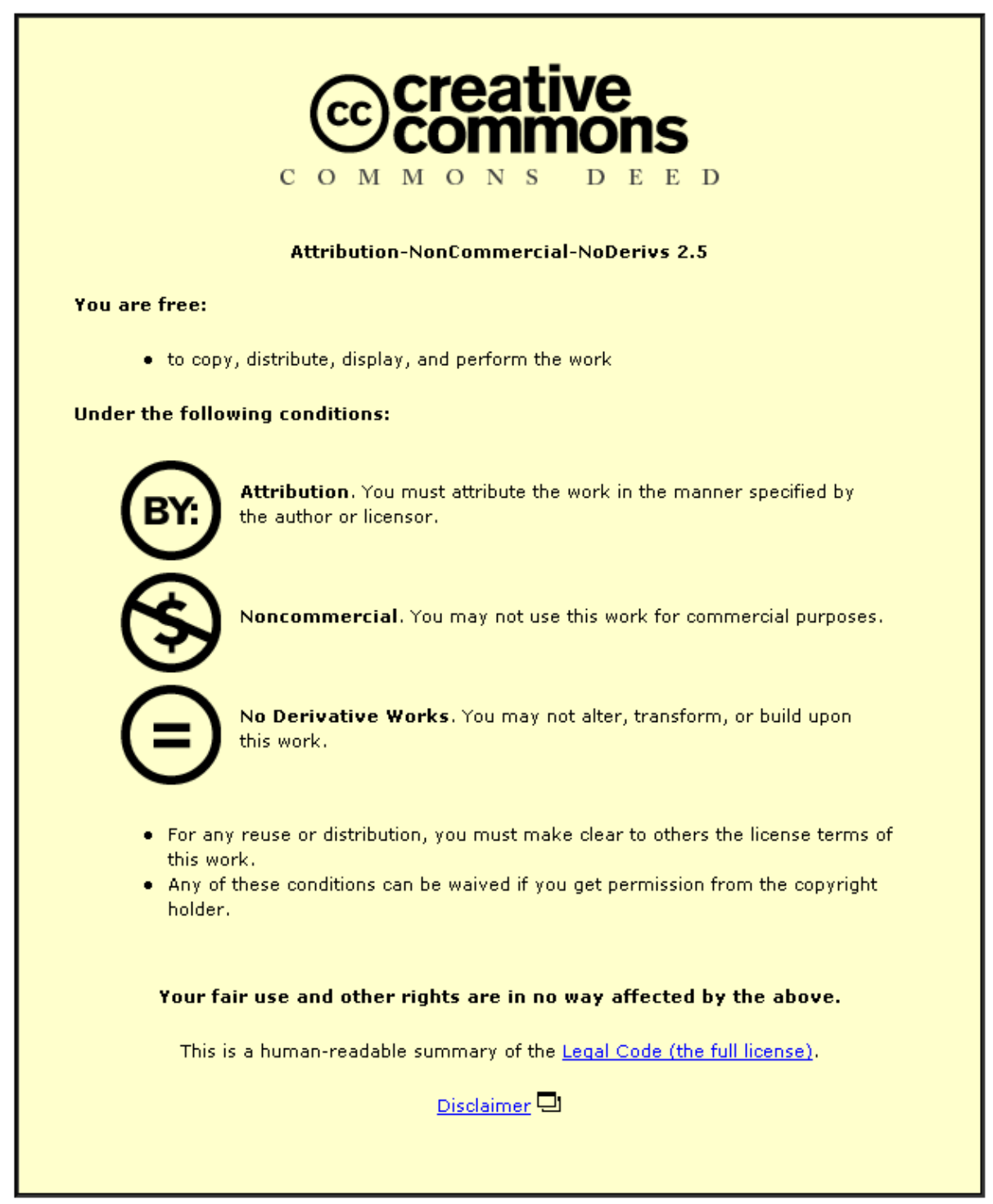

For the full text of this licence, please go to: http://creativecommons.org/licenses/by-nc-nd/2.5/ 


\title{
Cross Organisational Compatible Plans Generation Framework
}

\author{
Mohammad Saleem ${ }^{1}$, Paul W.H. Chung ${ }^{1}$, Shaheen Fatima ${ }^{1}$, Wei Dai ${ }^{2}$
}

\begin{abstract}
In this modern era, organisations have to work in coordination with many other organisations in order to succeed in business. Interacting organisations can only proceed in business if they have compatible workflows. This paper proposes a framework to automatically generate compatible workflows for multiple interacting organisations from their process definitions and service descriptions. Existing systems can reconcile existing workflows only, and cannot generate compatible workflows for multiple organisations automatically. The proposed system is different from existing systems since it targets workflow collaboration by generating workflows automatically. This allows the organisations to save the time that would otherwise be spent in modelling workflows and making them compatible with the workflows of interacting organisations.
\end{abstract}

\section{Introduction}

With the development of the Internet, there has been an increase in demand for business process automation. A business process is a set of ordered interlinked procedures and activities within the context of an organisational structure, which brings about an organisational goal [1]. When two or more organisations conduct business together, the need for cross organisational business process automation arises. Hence, there is a need for cross organisational workflow collaboration.

Business collaboration can only work if the workflows of the interacting organisations are compatible [2]. Interacting workflows are compatible when they have an agreed sequence of interface activities. An interface activity is a point where exchange of collaborative messages and information takes place between two in-

\footnotetext{
${ }^{1}$ Computer Science Department, Loughborough University, Loughborough, LE11 3TU, UK

\{M.Saleem, P.W.H.Chung, S.S.Fatima\}@lboro.ac.uk

${ }^{2}$ School of Management and Information Systems, Victoria University, Melbourne, Victoria, Australia
}

Wei.Dai@vu.edu.au 
teracting workflows [3]. Considerable effort is needed to ensure that workflows are compatible in the first place $[4,5]$.

Incompatibilities among workflows should be removed, through reconciliation, in order to proceed in business. If an organisation works with other organisations in coordination, this can be a highly time consuming task. To allow efficient use of time and resources, this paper proposes a new framework for cross organisational workflow generation and cross organisational workflow collaboration.

In the proposed framework, we treat the workflow generation problem as an AI planning problem [6]. The proposed system is implemented using Simple Hierarchical Ordered Planer 2 (SHOP2) [7, 8] for planning. SHOP2 requires domain description for planning. Domain description consists of operators and methods [9]. Operators are primitive tasks and methods are specifications for decomposing composite tasks. The proposed system translates process definitions of the interacting organisations to operators and methods, and uses the operators and methods to automatically create compatible plans for the interacting organisations. This enables the organisations to avoid the time consuming task of reconciliation.

Section 2 explains cross organisational domain compatible plans generation framework. Section 3 explains an example scenario for a customer and vendor, Section 4 summarizes related work. Conclusions are drawn in Section 5.

\section{Cross Organisational Compatible Plans Generation Framework}

Fig. 1 shows the proposed cross organizational compatible plans generation framework.

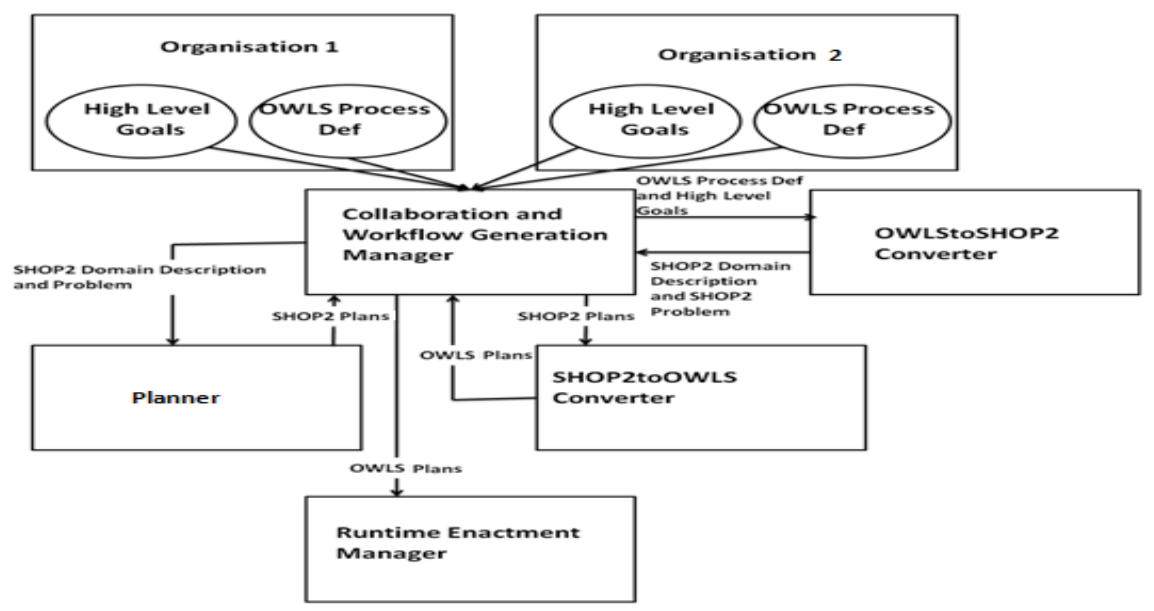

Fig. 1 Cross Organisational Compatible Plans Generation Framework. 
Although the proposed framework works for any number of interacting organisations, for clarity, the figure only depicts two. Each organization models its OWLS process definitions and passes them to Collaboration and Workflow Generation Manager (CWGM). The organisations select their high level goals on the GUI. CWGM passes the process definitions to OWLStoSHOP2 translator, which translates them into SHOP2 domain descriptions. OWLStoSHOP2 translator also translates high level goals into a SHOP2 problem.

CWGM removes all those operators from the domain descriptions that are not used in planning. CWGM also collapses domain descriptions of all interacting organisations into a single joint domain, and SHOP2 problems of all interacting organisations into a single joint SHOP2 problem. Pre-planning analysis of the joint domain and joint problem is done so that operators, inputs (pre-conditions) and outputs of interacting organisations can be tracked. Operators in the domain that can form parallel plans are identified. Based on identified operators, sub-methods are inserted into the joint domain description. The inserted sub-methods are used by SHOP2 to create all possible plans. The original algorithm of SHOP2 is modified to suit this functionality.

The joint SHOP2 problem and the joint SHOP2 domain are passed to SHOP2 planner which creates all possible joint plans. A joint plan is a combined plan of all interacting organisations which achieves the combined goals of all the organisations. A joint plan is subdivided to create a set of interacting plans, one plan for each organisation, compatible with each other.

The set of compatible plans with the least number of activities is highlighted to the interacting organisations for execution. The highlighted or any other selected set of compatible SHOP2 plans is transferred to SHOP2toOWLS converter to convert the plans from SHOP2 format into OWL-S format. OWL-S plans are further passed to Runtime Enactment Manager which executes actual WSDL services in the OWLS plans.

\section{Example}

We generate compatible plans for a vendor and customer interacting with each other as an example. The vendor is an overseas exporter while the customer is an overseas importer. Based on the process definitions of vendor and customer, twenty possible set of compatible plans that can reach the goal states of the interacting organisations are generated. Fig. 2 shows processes of the vendor and customer and two of the twenty sets of compatible plans generated.

In this paper, an activity name followed by " $s$ " or " $r$ " depicts sending and receiving collaboration messages or collaboration information respectively. 
Mohammad Saleem, Paul W.H. Chung, Shaheen Fatima and Wei Dai

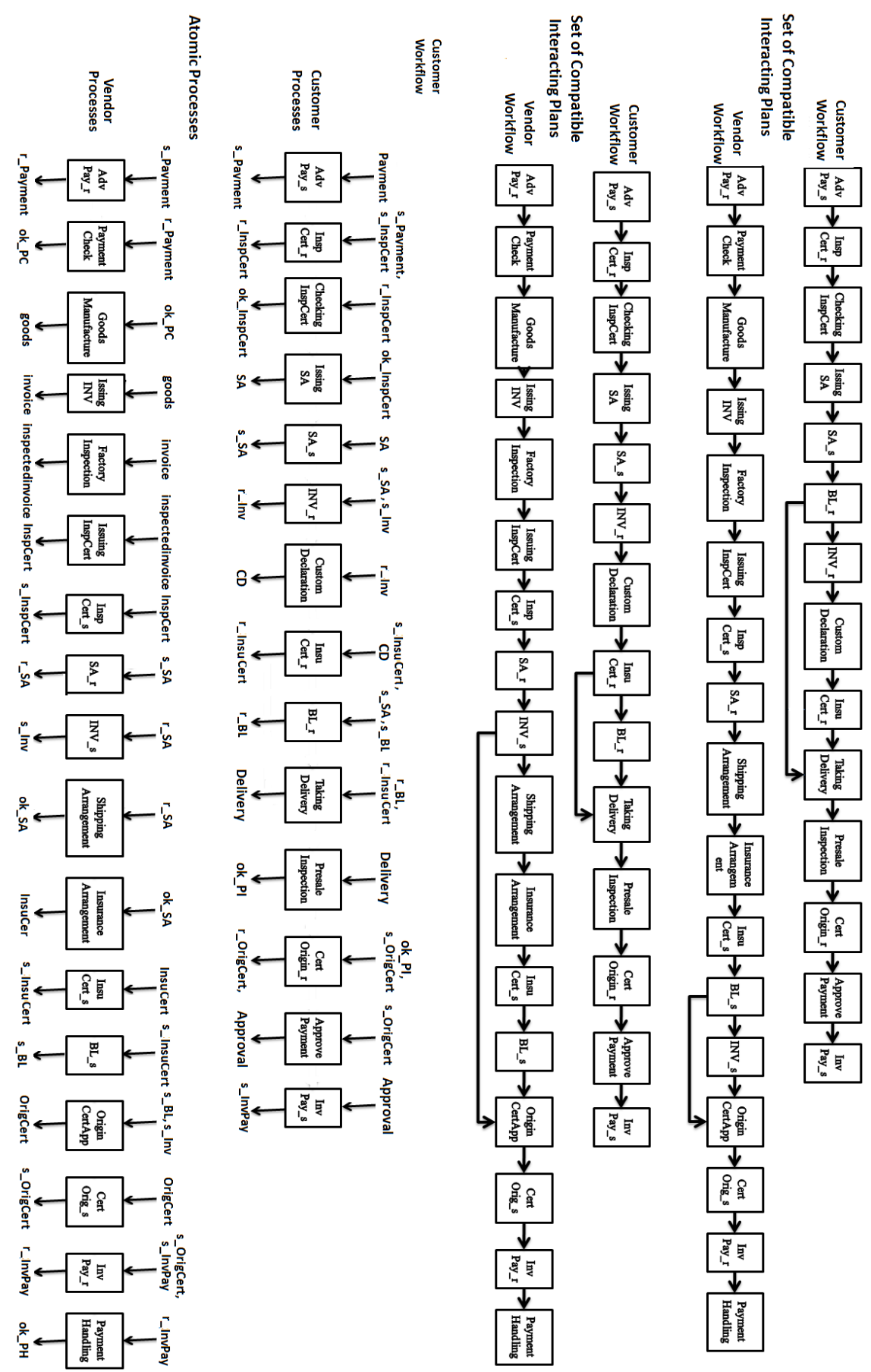

Fig. 2 Atomic Processes and Two Sets of Compatible Interacting Workflows. 


\section{Related Work}

Existing research on workflow collaboration has mostly focused on coordination between existing workflows. Chen and Chung [3] proposed a framework for cross-organizational workflow collaboration. The framework proposed by Chen and Chung compares existing workflows of two organisations, detects incompatibilities between the workflows and gives offers and counter offers to the interacting organisations to reach reconciliation. This framework saves time and resources for the organisations by automatically creating offers and counteroffer for workflow reconciliation. The organisations still have to model their workflows initially in order to collaborate. The organisations also have to invest time by either accepting or rejecting the automatically generated offers, every time the organisations collaborate with new organisations.

Sirin et al. [10] initially presented a web service composition system to compose web services semi-automatically. Sirin et al. [9] later extended the semiautomatic system into a fully automated system. Their proposed system uses SHOP2 for web services composition. Sirin et al. also proposed a sound and complete algorithm for translating OWL-S processes into SHOP2 domain description. The system proposed by Sirin et al. is able to create plans from OWL-S processes of a single user or single organisation. It does not take cross organisational collaboration into account. The system also does not generate all possible plans.

Transplan [11] is another system based on SHOP2 planning algorithm to generate multiple plans from OWLS process definitions. Transplan uses the translation algorithm put forward by Sirin et al. [9]. Transplan does not guarantee to create all possible plans. Transplan also does not target collaboration among multiple organisations.

Work done by Sirin et al. is closely related to the work reported in this paper since they use OWLS process definitions of the interacting organisations, translate them to SHOP2 domain descriptions and create SHOP2 plans from the domain descriptions. All systems discussed above either target workflow collaboration between existing workflows, or they focus on automatic workflow generation for a single organisation. Framework presented in this paper targets automatic workflow generation and cross organizational collaboration at the same time.

\section{Conclusion}

This paper proposes a framework for the generation of compatible workflows for multiple interacting organisations. The proposed framework is different from ex- 
isting systems since existing systems reconcile existing workflows. Other systems generate workflows for single organisations only and do not take cross organisational collaboration into account. The proposed framework targets the integration of workflow generation and workflow collaboration.

The proposed system generates all possible sets of compatible workflows for interacting organisations and highlights the least costly set of compatible workflows to the interacting organisations for execution. The aim in future is to develop a runtime enactment mechanism that enacts the actual WSDL services in the plan and makes sure that the transfer of information and files among the interacting organisations occur smoothly.

\section{Acknowledgments}

This project is being funded by the UK Engineering and Physical Sciences Research Council (EPSRC) through the Innovative Manufacturing and Construction Research Centre (IMCRC) at Loughborough University.

\section{References}

1. Workflow Management Coalition: Terminology \& Glossary. Technical Report WFMC-TC1011 (1999).

2. Yang J., Papazoglou M.: Interoperation Support for Electronic Business. Communication of the ACM, Vol. 43, No. 6, pp. 39-47 (2000).

3. Chen X., Chung P.W.H.: Facilitating B2B E-Business by IT-Supported Business Process Negotiation Services. In: Proceedings of the 2008 IEEE International Conference on Service Operations and Logistics and Informatics, pp. 2800-2805 (2008).

4. Schulz K., Orlowska M.: Facilitating cross-organizational workflows with a workflow view approach. Data and Knowledge Engineering, Vol. 51, No.1, pp. 109-147 (2004).

5. Chiu D.K.W., Cheung S.C., Karlapalem K., Li Q., Till S., Kafeza E.: Workflow View Driven Cross-Organizational Interoperability in a Web-Services Environment. Information Technology and Management, Vol. 5, pp. 221-250 (2004).

6. Dong X., Wild D.: An Automatic Drug Discovery Workflow Generation Tool using Semantic Web Technologies. In: Proceedings of Fourth IEEE International Conference on eScience, pp. 652-657 (2008).

7. Nau D., Mũnoz-Avila H., Cao Y., Lotem A., Mitchell S.: Total-order planning with partially ordered subtasks. In: IJCAI-2001, Seattle (2001).

8. Nau D., Au T., Ilghami O., Kuter U., Murdock J., Wu D., Yaman F.: SHOP2: An HTN planning system. Journal of Artificial Intelligence Research, pp. 379-404 (2003).

9. Sirin E., Parsia B., Wu D., Hendler J., Nau D.: HTN planning for web service composition using SHOP2. Journal of Web Semantics, Vol. 1, No. 4, pp. 377-396 (2004).

10. Sirin E., Hendler J., Parsia B.: Semi-automatic composition of Web services using semantic descriptions. In: Proceedings of Web Services: Modeling, Architecture and Infrastructure workshop in conjunction with ICEIS (2003).

11. Transplan. http://sourceforge.net/projects/transplan/ 